\title{
THz Oscillations in a GaN Based Planar Nano-Device
}

\author{
K. Y. Xu' ${ }^{1}$, Y. N. Wang' ${ }^{2}$ Z. N. Wang ${ }^{2}$ J. W. Xiong' ${ }^{2}$, G. Wang ${ }^{2}$ \\ ${ }^{1}$ Laboratory of Quantum Information Technology, School of Physics and Telecommunication Engineering, South China Normal \\ University, Guangzhou, China; ${ }^{2}$ State Key Laboratory of Optoelectronic Materials and Technologies, Sun Yat-sen University, \\ Guangzhou, China. \\ Email:xuky@scnu.edu.cn
}

Received September 2013

\begin{abstract}
Gunn oscillations in a GaN based planar nano-device have been studied by ensemble Monte Carlo (EMC) method. Simulation results show that when the channel length of the device reduces to $450 \mathrm{~nm}$, THz oscillations (about $0.3 \mathrm{THz}$ ) can be obtained. Also the phase of the oscillations can be controlled by the initial conditions that excite the Gunn domains. Moreover, through adjusting the phase difference between the oscillations in a double-channels device, which attained by parallel connecting two single-channel devices, the frequency of the device shifts from $0.3 \mathrm{THz}$ to $0.6 \mathrm{THz}$. This phenomenon remains in devices with shorter channel-length, unless the channel-length is too short to support Gunn oscillations. The possible underlying mechanisms are also discussed.
\end{abstract}

Keywords: THz Oscillation; Gunn; Monte Carlo; GaN; Planar Nanodevice

\section{Introduction}

The terahertz $(1 \mathrm{THz}=1000 \mathrm{GHz})$ electromagnetic wave has attracted wide attentions as it could enable very broad applications ranging from non-destructive imaging and spectroscopy of biological materials, remote detection of hidden objects and explosives, to manipulations of quantum states in semiconductors [1,2]. Moreover, development of semiconductor $\mathrm{THz}$ electronic devices is also certainly paramount and timely to future generation of largevolume information processing and high-performance computations. However, the development of $\mathrm{THz}$ technology is so far largely hampered by the lack of reliable, solid-state sources operating at room-temperature [3].

One possible way of developing $\mathrm{THz}$ devices is to adapt well-known mechanisms that have been already utilized in microwave field for higher frequency applications. Among them, Gunn Effect in GaN is considered as one of the most promising candidates for further $\mathrm{THz}$ sources, which has attracted wide attention recently [4-8].

Heat dissipation is considered as one of the most challenging issues for practical GaN-based Gunn sources. To conquer this problem, planar devices, such as SSDs [9], may be a suitable choice, since planar architecture allows easier design for heat dissipation $[5,8]$. In addition, in planar devices the electrodes are connected side by side to the active semiconductor layer rather than placed on top of each other, as in conventional multilayered verticalstructured devices, resulting in very low parasitic capa- citances. As such, high speeds are attainable.

In this work, we focus on studying the behaviors of Gunn oscillations in GaN based SSDs at room temperature. SSDs as shown in Figure 1 are planar nanodevices, which not only have high operation speed, up to $\mathrm{THz}$ at room temperature, but also benefit for the propagation of Gunn domains [10]. The paper is structured as follows. In Section 2, device structures and simulation models are introduced. In Section 3, the time-dependent Gunn oscillations are studied under different bias conditions. Then the relationship between the initial phase of the oscillation and the exciting condition is established. And moreover the interaction of Gunn oscillations in adjacent double channels is further studied under different phase conditions. In Section 4, simulation results are further discussed.

\section{Device Structures and EMC Model}

Figure 1(a) shows schematically the top view of a double-channels device including two SSDs and will be mentioned as D-SSD in follows. The device is based on a GaN/AlGaN heterostructure, where a 2DEG is formed at the hetero-interface with a carrier concentration of $8.0 \times$ $10^{12} \mathrm{~cm}^{-2}$ [8]. For a single-channel SSD (S-SSD) framed by green dashed line in Figure 1(a), the two L-shaped insulating trenches are etched through the 2DEG layer, which ensures that electrons have to pass the narrow channel between the two trenches in order to conduct a 
(a)
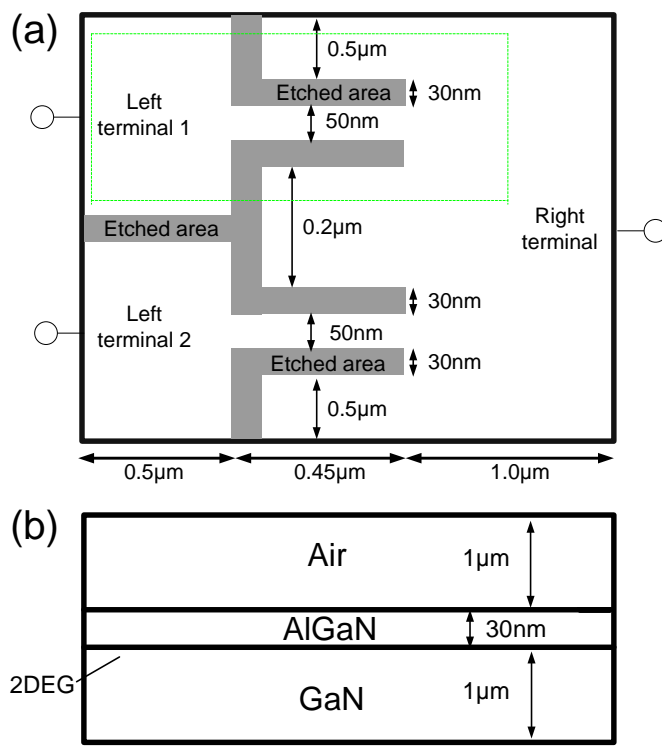

Figure 1. Schematic top view (a) and side view (b) of the simulated T-SSD (not to scale). The gray areas and the white areas in the top view represent 2DEG and insulating trenches, respectively. The green dash-lines delineate the typical structure of single SSDs. An interface of AlGaN/ GaN heterostructures in (b) is just $30 \mathrm{~nm}$ bellows the device surface, at which a sheet of 2DEG forms.

current between the left and right terminals. The two S-SSDs are both designed with a channel width of $50 \mathrm{~nm}$ and a trench width of $30 \mathrm{~nm}$. Other geometric parameters are defined in Figure 1(a). In the D-SSD, the two SSSDs have separated left terminals, but share the same right terminal. The design of sharing the same right terminal will benefit the interactions between channels, and that of using different left terminals will entitle us to control the Gunn oscillations through applied voltages.

In order to obtain the operation properties of the devices, a semi-classical 2D EMC method self-consistently coupled with 3D Poisson equations is used here. This 2D-3D combined model is developed from our entirely 2D EMC model, which has been used in earlier work [10-13]. Details of the above 2D-3D combined model and the comparison with fully 3D EMC method $[14,15]$, can be found in our recent work [16]. Figure 1(b) shows schematically the side view of the simulated SSDs. In order to properly include the 3D electric-field coupling, Poisson equations are solved in a domain beyond the geometric structure of the SSDs. As one can find from Figure 1(b), a volume with a height of one micrometer above the device surface is also included. The dielectric constant used in the simulations for Air, AlGaN and GaN are 1, 8.5 and 8.9, respectively. In order to model the influence of surface states at the semiconductor-air interface, a uniform negative charge density, $0.8 \times 10^{12} \mathrm{~cm}^{-2}$, is also added at the edge of the insulating trenches during the simulations. All simulations are carried out at room temperature.

\section{Simulation Results}

In this section, EMC method is used to study the Gunn oscillations in S-SSDs and D-SSDs. Simulations are firstly carried out on S-SSDs under different bias condition to study the voltage dependence and the initial phase of Gunn oscillations. Then the interaction of Gunn oscillations is further studied in D-SSDs under different phase conditions.

\subsection{Single-Channel Devices}

For S-SSD simulations, the left terminal of the device is always grounded and the current output from the right terminal is recorded. Figure 2 shows the time-dependent current output of an S-SSD when a serial of step voltages are applied on the right terminal. A current peak with sharp rise and fall is shown after each abrupt change of the applied voltage, which is anticipated as a result of the charging of parasitic capacitances in the device [10]. This parasitic-capacitance-induced current-peak will be mentioned as PCIC peak in the following studies. The current after each PCIC peak increases with the applied voltage and shows obvious oscillation behaviors when the applied voltage beyond $16 \mathrm{~V}$. Since the length of the channel is $450 \mathrm{~nm}$, the threshold electric field for Gunn oscillation would be about $0.4 \mathrm{MV} / \mathrm{cm}$, which agrees with recent experimental results [4]. Recently, by using an entirely 2D EMC model, SSDs with a wider channelwidth have been studied and a special mode with two domains simultaneously forming in the channel have been found, but the mechanism is still puzzled [8]. The period of the oscillations shown in Figure 2 is about 3 ps, corresponding to frequency about $0.3 \mathrm{THz}$. The period of the oscillations is also the time for Gunn domain to travel through the channel, so that the drift velocity of the Gunn domain should be about $1.5 \times 10^{7} \mathrm{~cm} / \mathrm{s}$, which is close to the saturated velocity of the electrons in $\mathrm{GaN}$ [17]. This result reveals that in our case, like those in traditional Gunn diodes, only one effective domain simultaneously exists.

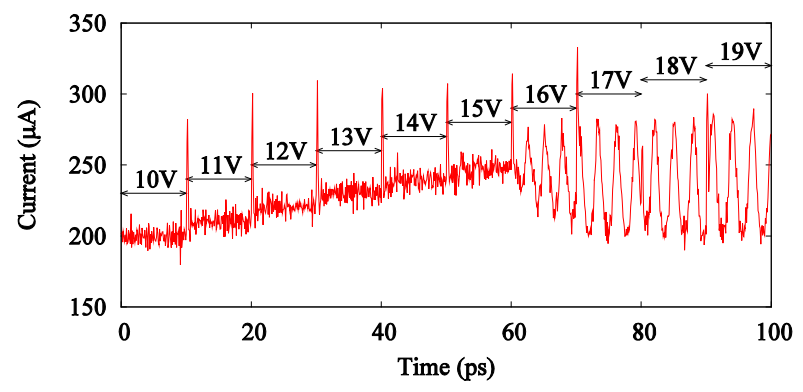

Figure 2. Current responses of a single SSD showed in Figure 1 under the application of a series voltage steps. 


\subsection{Phase Control}

Further investigations tell us that the initial phase of the Gunn oscillations is dependent on what time the step voltage is applied. If the time interval of two step voltages is $\mathrm{T}_{i}$, the phase-shift between the corresponding excited-oscillations will be $2 \pi \mathrm{T}_{i} / \mathrm{T}$, where $\mathrm{T}$ is the period of the oscillations. Typical results are shown in Figure 3. The applied step voltages change their values from $0 \mathrm{~V}$ to $21 \mathrm{~V}$ at 2 ps and 3.5 ps respectively, resulting in two PCIC peaks. Since these PCIC peaks are too strong and with non-needing information for our topic, only parts of them are shown in Figure 3. One can find that the phase of the two oscillations is opposite, because the time interval is just half of the oscillation period.

\subsection{Double-Channel Devices}

During D-SSD simulations, the right terminal is grounded and two different negative step voltages are applied to the two left terminals, respectively. Results for special time interval $\mathrm{T}_{i}=0 \mathrm{ps}$ and $\mathrm{T}_{i}=1.5 \mathrm{ps}$ are shown in Figure 4. PCIC peaks are also not shown in whole as those in Figure 3. The time-dependent output-current induced by step voltages with zero time-interval is similar to that in S-SSD. However, that induced by step voltages with 1.5 ps interval shows obvious second-harmonic oscillation with period of about 1.5 ps, just half of that in SSSD, corresponding to a frequency of about $0.6 \mathrm{THz}$.

The distinct deviation of current output between in phase and out phase oscillations may come from the following facts. The two channels are identified, so the potential distributions along the two channels should be the same for the in-phase case. But for the out-phase case, the potential distributions must be different and might also changes with time. As a result, in the out-phase case, the two oscillations will mutually modulate. For simplification, the Gunn oscillations can be assumed to obey sinusoidal function with frequency of $\mathrm{f}$, so that the mutual modulation could be reasonably described with the same sinusoidal function. Consequently, the current output would be proportional to the square of the sinusoidal function, which possesses a frequency of $2 \mathrm{f}$.

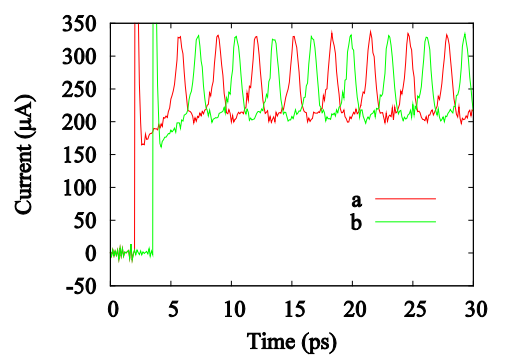

Figure 3. Current responses of a single SSD showed in Figure 1 when the applied voltage changes from 0 to $21 \mathrm{~V}$ at (a) 2 ps and (b) 3.5 ps.

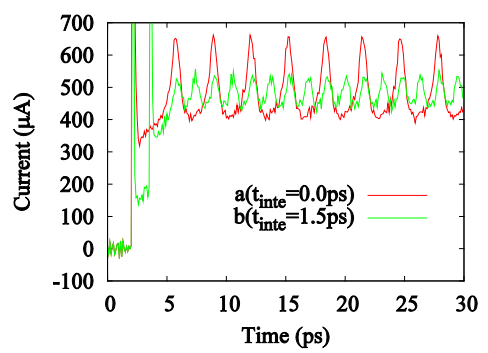

Figure 4. Current responses of a T-SSD showed in Figure 1 for (a) without and (b) with self-gating effect.

It is well known that when two oscillators are put together, they would show synchronized oscillations [18]. As such, more simulations are carried out for D-SSD in the out-phase case to examine the persistence of the above modulations. Results show that even in a much longer simulation time (longer than 300 ps which including at least 600 periods of oscillations), the waveform of the current output does not change. Moreover, further investigations show that the above phenomenon still exists in shorter-channel D-SSDs so that oscillations with higher frequency can be obtained. For an instance, when the channel length is reduced to $300 \mathrm{~nm}$, the corresponding frequency is raised to about $1 \mathrm{THz}$, results not shown here.

\section{Conclusions}

In conclusions, Gunn oscillations in GaN based S-SSDs and D-SSDs have been studied by ensemble Monte Carlo (EMC) method in detail. We show that since the channel length of the device is extremely short (only $450 \mathrm{~nm}$ ) the oscillations in S-SSDs operate with a frequency about 0.3 THz. Also the initial phase of Gunn oscillations can be controlled by applied voltage. Moreover, for D-SSDs, by adjusting the phases of two oscillations in each channel to be contrary, the frequency of Gunn oscillation can be increased from $0.3 \mathrm{THz}$ to $0.6 \mathrm{THz}$. This phenomenon may attribute to the mutual modulations between the two oscillations and occurs even in shorter channels so long as Gunn oscillation can occur, resulting in oscillations with frequency up to $1 \mathrm{THz}$.

\section{Acknowledgements}

This work was supported by FOK YING TONG Education Foundation (No. 122004), Natural Science Foundation of Guangdong Province, China (No. S2013010012711) and NSFC (Grands U0934002).

\section{REFERENCES}

[1] M. Sherwin, “Terahertz Power,” Nature, Vol. 420, 2002, pp. 131-133. http://dx.doi.org/10.1038/420131a

[2] M. Tonouchi, "Cutting-Edge Terahertz Technology," 
Nature Photonics, Vol. 1, 2007, pp. 97-105. http://dx.doi.org/10.1038/nphoton.2007.3

[3] P. H. Siegel, “Terahertz Technology,” IEEE Transactions on Microwave Theory Technology, Vol. 50, No. 3, 2002, pp. 910-928. http://dx.doi.org/10.1109/22.989974

[4] N. Ma, B. Shen, F. J. Xu, L. W. Lu, Z. H. Feng, Z. G. Zhang, S. B. Dun, C. P. Wen, J. Y. Wang, F. Lin, D. T. Zhang and M. Sun, "Current-Controlled Negative Differential Resistance Effect Induced by Gunn-Type Instability in n-Type GaN Epilayers,” Applied Physical Letters, Vol. 96, 2010, Article ID: 242104. http://dx.doi.org/10.1063/1.3455070

[5] A. Íñiguez-de-la-Torre, I. Íñiguez-de-la-Torre, J. Mateos and T. González, "Correlation between Low-Frequency Current-Noise Enhancement and High-Frequency Oscillations in GaN-Based Planar Nanodiodes: A Monte Carlo Study,” Applied Physical Letters, Vol. 99, 2011, Article ID: 062109. http://dx.doi.org/10.1063/1.3613956

[6] Y. Hao, J. F. Zhang, B. Shen and X. Y. Liu, "Progress in Group III Nitride Semiconductor Electronic Devices,” $J$. Semicond., Vol. 33, No. 8, 2012, Article ID: 081001. http://dx.doi.org/10.1088/1674-4926/33/8/081001

[7] L. A. Yang, S. Long, X. Guo and Y. Hao, “A Comparative Investigation on Sub-Micrometer InN and GaN Gunn Diodes Working at Terahertz Frequency," Journal of Applied Physics, Vol. 111, 2012, Article ID: 104514. http://dx.doi.org/10.1063/1.4721667

[8] A. Íñiguez-de-la-Torre, I. Íñiguez-de-la-Torre, J. Mateos, T. González, P. Sangaré, M. Faucher, B. Grimbert, V. Brandli, G. Ducournau and C. Gaquière, "Searching for $\mathrm{THz}$ Gunn Oscillations in GaN Planar Nanodiodes," Journal of Applied Physics, Vol. 111, 2012, Article ID: 113705. http://dx.doi.org/10.1063/1.4724350

[9] A. M. Song, M. Missous, P. Omling, A. R. Peaker, L, Samuelson and W. Seifert, "Unidirectional Electron Flow in a Nanometer-Scale Semiconductor Channel: A Self-Switching Device,” Applied Physical Letters, Vol. 83, 2003, p. 1881. http://dx.doi.org/10.1063/1.1606881

[10] K. Y. Xu, G. Wang and A. M. Song, "Gunn Oscillations in a Self-Switching Nanodiode,” Applied Physical Letters,
Vol. 93, 2008, Article ID: 233506. http://dx.doi.org/10.1063/1.3042268

[11] K. Y. Xu, X. F. Lu, G. Wang and A. M. Song, "Strong Spatial Dependence of Electron Velocity, Density, and Inter-Valley Scattering in an Asymmetric Nanodevice in the Nonlinear Transport Regime,” IEEE Transactions on Nanotechnology, Vol. 7, No. 4, 2008, pp. 451-457. http://dx.doi.org/10.1109/TNANO.2008.926348

[12] K. Y. Xu, X. F. Lu, G. Wang and A. M. Song, "Enhanced Terahertz Detection by Localized Surface Plasma Oscillations in a nanoscale unipolar diode,” Journal of Applied Physics, Vol. 103, 2008, Article ID: 113708. http://dx.doi.org/10.1063/1.2937175

[13] K. Y. Xu, X. F. Lu, A. M. Song and G. Wang, “Terahertz Harmonic Generation Using a Planar Nanoscale Unipolar Diode at Zero Bias,” Applied Physical Letters, Vol. 92, 2008, Article ID: 163503. http://dx.doi.org/10.1063/1.2907490

[14] T. Sadi, F. Dessenne and J.-L. Thobel, “Three-Dimensional Monte Carlo Study of Three-Terminal Junctions Based on InGaAs/InAlAs Heterostructures," Journal of Applied Physics, Vol. 105, 2009, Article ID: 053707. http://dx.doi.org/10.1063/1.3087703

[15] T. Sadi and J.-L. Thobel, "Analysis of the High-Frequency Performance of InGaAs/InAlAs Nanojunctions Using a Three-Dimensional Monte Carlo Simulator," Journal of Applied Physics, Vol. 106, 2009, Article ID: 083709. http://dx.doi.org/10.1063/1.3248358

[16] K. Y. Xu, J. W. Xiong, A. M. Song and G. Wang, "Effects of Three-Dimensional Electric-Field Coupling on a Side-Gated Nanotransistor,” Semicond. Sci. Technol., Vol. 26, No. 9, 2011, Article ID: 095026. http://dx.doi.org/10.1088/0268-1242/26/9/095026

[17] S. Chen and W. Gang, "High-Field Properties of Carrier Transport in Bulk Wurtzite GaN: A Monte Carlo Perspective," Journal of Applied Physics, Vol. 103, 2008, Article ID: 023703. http://dx.doi.org/10.1063/1.2828003

[18] B. Razavi, "Mutual Injection Pulling between Oscillators," IEEE 2006 Custom Integrated Circuits Conference (CICC), California, 10-13 September 2006, pp. 675-678. 